\title{
Studies of jet production properties and the strong coupling constant with the ATLAS detector
}

\author{
Pavel Starovoitov* on behalf of the ATLAS Collaboration \\ Kirchhoff-Institut für Physik \\ Im Neuenheimer Feld, 227 \\ 69120 Heidelberg, Germany \\ E-mail: pavel.starovoitovecern.ch
}

\begin{abstract}
Several aspects of jet production in proton-proton collisions have been measured by the ATLAS
Collaboration. The measurements of the jet production cross-sections probe the dynamics of QCD and can constrain the parton proton structure. Cross-sections for inclusive jet, three-jet and four-jet final states are measured differentially in a variety of kinematic variables and for different centre-of-mass energies in proton-proton collisions with the ATLAS detector. Experimental results are compared to expectations based on next-to-leading order QCD calculations as well as to the Monte Carlo simulations. First LHC Run-2 results are also presented. The measurement of the jet-jet transverse energy correlations sensitive to the strong coupling constant is discussed. The momentum-weighted sum of the charges of tracks associated to a jet is sensitive to the electrical charge of the parton initiating the jet. The distribution of the so-called jet charge has been measured in dijet events using proton-proton collision data.
\end{abstract}

The European Physical Society Conference on High Energy Physics

22-29 July 2015

Vienna, Austria

${ }^{*}$ Speaker. 


\section{Introduction}

At the Large Hadron Collider (LHC) [1], jet production in proton-proton (pp) collisions can be used to probe quantum chromodynamics (QCD) in the $\mathrm{TeV}$ regime. In $\mathrm{QCD}$, jet production can be interpreted as the fragmentation of quarks and gluons produced in the scattering process and its measurement provides information about the colour-exchange interaction. Therefore, the measurement of the multi-jet production cross-sections at different centre-of-mass energies at the LHC provides valuable inputs to the determination of the parton distribution functions (PDFs) in the proton and the strong coupling constant $\left(\alpha_{s}\right)$.

Jets are identified using the anti- $k_{t}$ algorithm [2] as implemented in the FastJet [3] software package. Inputs to the jet algorithm can be partons in the QCD calculation, stable particles after the hadronisation process in the Monte Carlo simulations or energy deposits in the detector.

Event shape variables, like transverse energy-energy correlations (TEEC), i.e. the energyweighted angular distributions of hadron jets produced in pp collisions, exhibit quadratic dependence on the strong coupling constant and can be used for precise $\left(\alpha_{s}\right)$ determination.

Quarks and gluons produced in high-energy particle collisions hadronise before their electric charge can be directly measured. However, information about the electric charge is embedded in the properties of hadron jets. The momentum-weighted charge sum of charged-particle tracks in a jet, jet charge, is sensitive to the electric charge of quarks and gluons. The jet charge is used in many applications, like heavy flavour quarks tagging [4], quark/gluon discrimination [5], etc.

In this contribution, the measurement of the double-differential inclusive jet production crosssection [6], the double-differential three-jet mass distribution [7], transverse energy-energy correlation [8] in pp collisions at centre-of-mass energy $\sqrt{s}=7 \mathrm{TeV}$, four-jet production cross-section [9] and jet charge distribution [10] in pp collisions at $\sqrt{s}=8 \mathrm{TeV}$ and the inclusive jet production cross-section [11] in pp collisions at $\sqrt{s}=13 \mathrm{TeV}$ using data collected by the ATLAS experiment [12] in 2011, 2012 and 2015 are presented.

\section{Inclusive jet and three-jet production cross-section measurements in proton-proton collisions at centre-of-mass energy $\sqrt{s}=7 \mathrm{TeV}$}

The double-differential inclusive jet cross-section is measured as a function of the transverse momentum of the jets, $p_{\mathrm{T}}$, and their absolute rapidity, $|y|$, at $\sqrt{s}=7 \mathrm{TeV}$ using the data corresponding to an integrated luminosity of $4.5 \mathrm{fb}^{-1}$. The measurement is performed using jets with $p_{\mathrm{T}}>100 \mathrm{GeV}$ and $|y|<3$. The measurements are made in five bins of $|y|$, in equal steps of 0.5 .

Events containing at least three jets within the $|y|<3$ with $p_{\mathrm{T}}>50 \mathrm{GeV}$ are considered for the double-differential three-jet mass distribution measurement. The leading, subleading and sub-subleading jets are required to have $p_{\mathrm{T}}>150 \mathrm{GeV}, p_{\mathrm{T}}>100 \mathrm{GeV}$ and $p_{\mathrm{T}}>50 \mathrm{GeV}$, respectively. The three-jet cross-sections are measured as a function of the three-jet mass $m_{j j j}=$ $\sqrt{\left(p_{1}+p_{2}+p_{3}\right)^{2}}$ and the summed absolute rapidity separation of the three leading jets $\left|Y^{*}\right|=$ $\left|y_{1}-y_{2}\right|+\left|y_{2}-y_{3}\right|+\left|y_{1}-y_{3}\right|$, where $p_{i}\left(y_{i}\right)$ are the four-momenta (rapidities) of the three leading jets. The measurements are made in five ranges of $\left|Y^{*}\right|<10$, in equal steps of two.

The uncertainty in the jet energy scale (JES) calibration is the dominant uncertainty in these measurements. The uncertainty in the $p_{\mathrm{T}}$ of each individual jet due to the JES calibration is between 

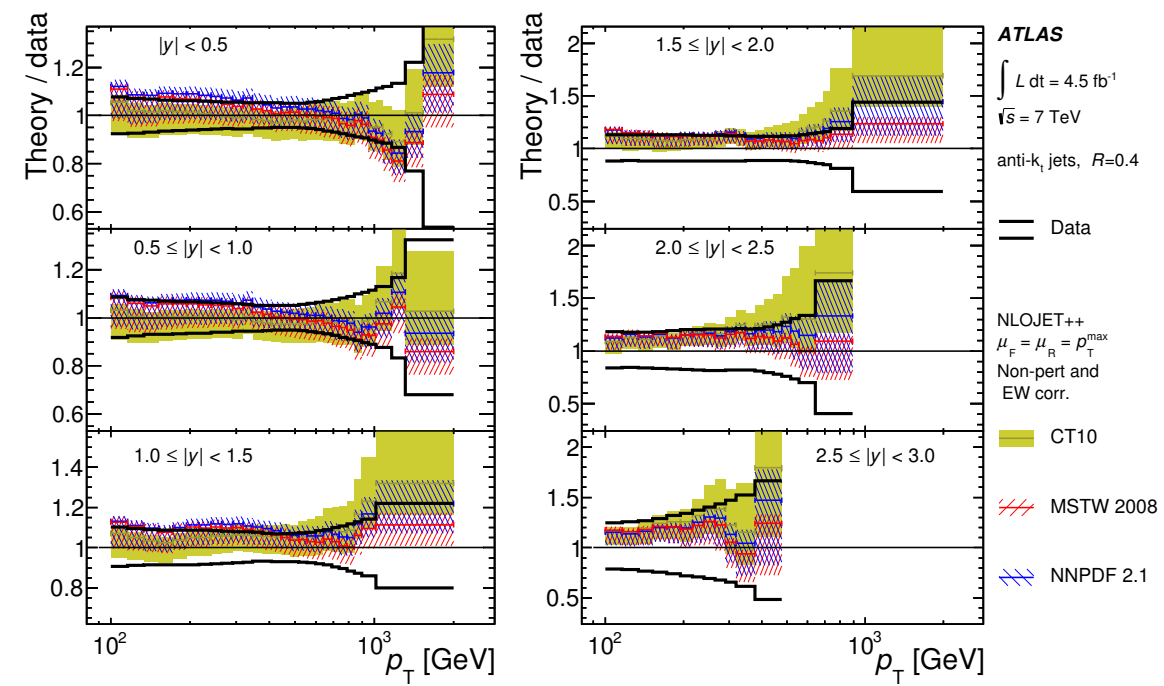

Figure 1: Ratio of NLO pQCD predictions to the measured double-differential inclusive jet cross-section [6], shown as a function of the jet $p_{\mathrm{T}}$ in bins of the $|y|$, for anti- $k_{t}$ jets with $R=0.4$.

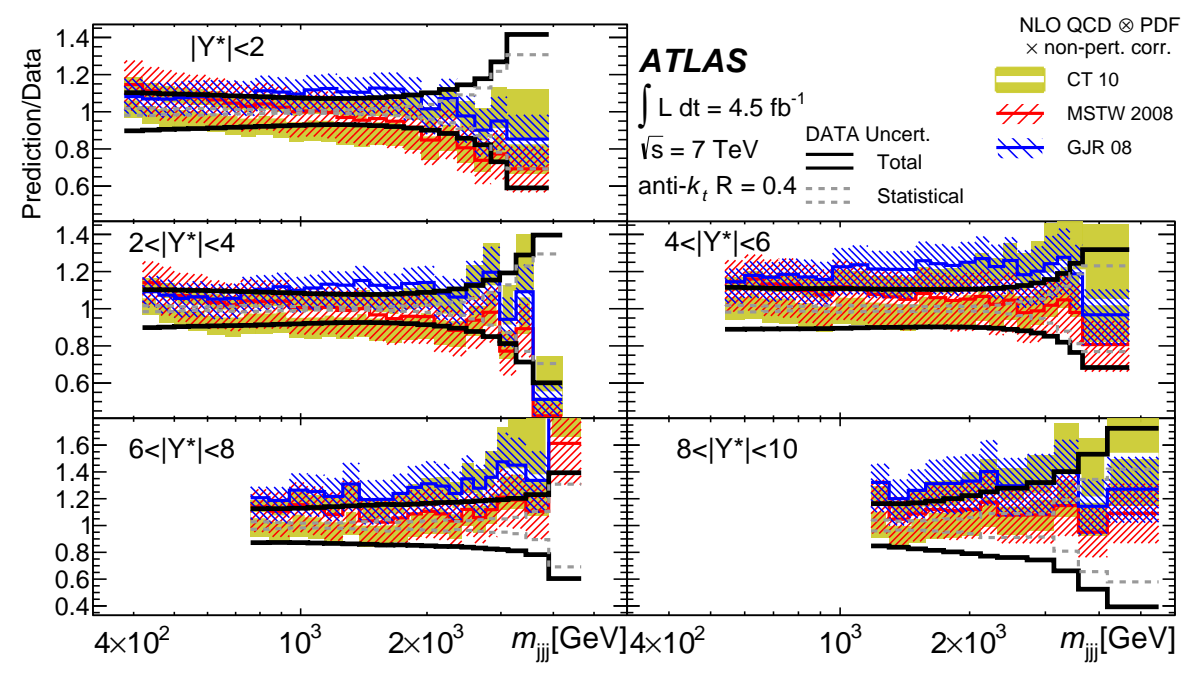

Figure 2: The ratio of NLO QCD predictions to data [7] as a function of $m_{j j j}$ in bins of $\left|Y^{*}\right|$ for jets identified using the anti- $k_{t}$ algorithm with $R=0.4$.

$1 \%$ and $4 \%$ in the central region $(|y|<1.8)$, and increases to $5 \%$ in the forward region $(1.8<|y|<$ 4.5). The uncertainties due to the JES calibration are propagated to the measured cross-sections using the MC simulation. The uncertainties due to the jet energy resolution (JER) and jet angular resolution (JAR) are propagated to the measured cross-section using the data unfolding procedure.

The next-to-leading order (NLO) predictions by the cross-section calculator NLOJET++ [13], corrected for hadronisation effects and underlying-event activity using PYTHIA 6 [14] Monte Carlo simulation with PERUGIA 2011 tune [15], are compared to the measured inclusive jet and three-jet cross-sections, as shown in Fig. 1 and 2, respectively. To estimate the uncertainty due to missing higher-order terms in the fixed-order perturbative expansion, the renormalisation and factorisation 
scales are independently varied up and down by a factor of two. The multiple uncertainty components of each PDF set, as provided by the various PDF analyses, are also propagated through the theoretical calculations. The uncertainties in the cross-sections due to the strong coupling are estimated using two additional proton PDF sets, for which different values of $\alpha_{s}$ are assumed in the fits, such that the effect of the strong coupling value on the PDFs is included. Non-perturbative corrections are evaluated using leading-logarithmic parton-shower generators. The corrections are calculated as bin-by-bin ratios of the cross-sections at the particle level, including hadronisation and underlying-event effects, to that at parton-level after the parton shower (before the hadronisation process starts) with the underlying event simulation switched off.

The inclusive jet measurement extends over jet $p_{\mathrm{T}}$ from $100 \mathrm{GeV}$ to $2 \mathrm{TeV}$ in the rapidity region $|y|<3$. The QCD predictions reproduce the measured cross-sections, which range over eight orders of magnitude in the six rapidity bins. The three-jet measurements probes the $m_{j j j}$ up to $\sim 5 \mathrm{TeV}$ and are well described by the calculations using various PDF sets.

\section{Transverse energy-energy correlation and strong coupling determination in events with at least two jets in proton-proton collisions at centre-of-mass energy $\sqrt{s}=7 \mathbf{T e V}$}

The data used in this analysis were recorded in 2011 at $\sqrt{s}=7 \mathrm{TeV}$ and correspond to an integrated luminosity of $158 \mathrm{pb}^{-1}$. The selected events must have at least two jets with transverse momentum $p_{\mathrm{T}}>50 \mathrm{GeV}$ and rapidity $|y|<2.5$. The two leading jets are further required to fulfil $p_{\mathrm{T}}^{1}+p_{\mathrm{T}}^{2}>500 \mathrm{GeV}$.

The transverse energy-energy correlation (TEEC) distribution for a sample of $N$ events is obtained by calculating the cosines of the angles in the transverse plane between all possible pairs of jets in each event, $\phi_{i j}$. Every pair $(i, j)$ represents an entry in the distribution, which is then weighted with the normalised product of the transverse energies, namely:

$\frac{1}{\sigma} \frac{d \Sigma}{d \cos \phi}=\frac{1}{N \Delta \cos \phi} \sum_{1}^{N} \sum_{i j} \frac{E_{T_{i}}^{A} E_{T_{j}}^{A}}{\left(\sum_{k} E_{T_{k}}^{A}\right)^{2}} \delta\left(\cos \phi-\cos \phi_{i j}\right)$

The data are corrected to the particle level in order

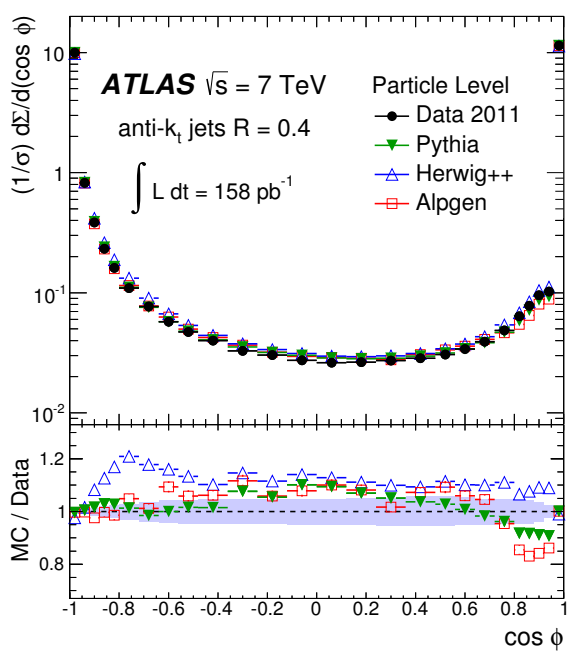

Figure 3: The unfolded distributions for transverse energy-energy correlation [8] along with comparisons to the MC expectations. The statistical uncertainties are shown with error bars, while the total experimental uncertainties are shown in a shaded band.

to take into account detector efficiencies and resolutions. This allows a direct comparison with theoretical calculations, as well as with measurements of other experiments.

The following experimental sources of uncertainty are considered for this measurement: JES, JER, JAR, pileup, parton shower modelling, unfolding procedure. The total experimental uncertainty is about 5\%, dominated by the JES and parton shower modelling components. 
The comparison of the measured TEEC spectrum to the MC predictions are shown in Figure 3. The description of the TEEC is good in the back-to-back region $\cos \phi \approx-1$ for both PYTHIA 6 [14] and AlPGEN+HeRWIg [16]. Differences up to $10 \%$ are observed in the central part, while the region of small angles shows differences as large as about $15 \%$. The description by HeRwIG++ [17] is poorer.

The NLO QCD calculations are performed using NLOJET++ [13]. These calculations are subsequently corrected for non-perturbative effects such as hadronisation and the underlying event (cf. Section 2). These calculations are used to determine the value of the strong coupling $\alpha_{s}\left(m_{Z}\right)$. The values of the strong coupling constant are extracted using several sets of PDF and the one obtained using the CT10 PDF [18] is chosen to be the final result for the $\alpha_{s}$ extraction, since its PDF uncertainty is largest and serves as an envelope covering the variations observed with different PDF sets.

The strong coupling constant determined from the measured TEEC distribution

$$
\alpha_{s}=0.1173 \pm 0.0010(\exp )_{-0.0020}^{+0.0063}(\text { scale }) \pm 0.0017(\mathrm{PDF}) \pm 0.0002(\mathrm{NPC})
$$

agrees very well with the world average value. Current determination is limited by the uncertainties due to the choice of renormalisation and factorisation scales.

\section{Four-jet production cross-section measurements in proton-proton collisions at centre-of-mass energy $\sqrt{s}=8 \mathrm{TeV}$}

This analysis studies events where at least four jets are produced in a hardscatter process. These events are of particular interest as the corresponding Feynman diagrams require several vertices even at leading order (LO) in the strong coupling constant $\alpha_{s}$. Cross-sections are calculated for events with at least four jets within the rapidity range $|y|<2.8$. Out of those four jets, the leading one must have $p_{\mathrm{T}}>$ $100 \mathrm{GeV}$, while the next

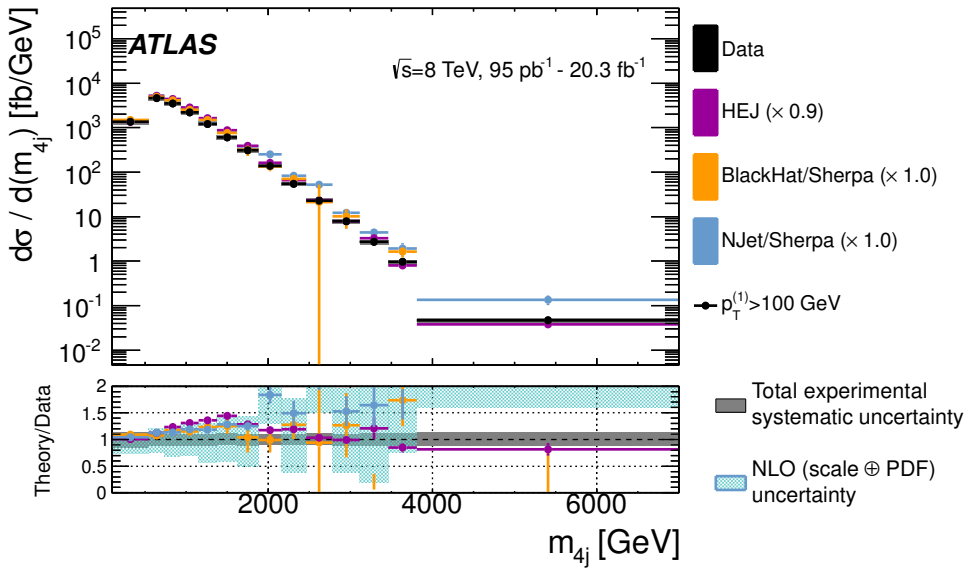

Figure 4: Unfolded four-jet differential cross-section as a function of $m_{4 j}$ [9], compared to different theoretical predictions. The solid band represents the total experimental systematic uncertainty centred at one. The patterned band represents the NLO scale and PDF uncertainties calculated from NJet/Sherpa centred at the nominal NJet/Sherpa values. three must have $p_{\mathrm{T}}>64 \mathrm{GeV}$. In addition, these four jets must be well separated from one another, such that the minimal angular distance between any pair of four leading jets $\Delta R=\sqrt{\Delta y^{2}+\Delta \phi^{2}}>$ 0.65. Cross-sections are measured differentially as a function of $m_{4 j}=\sqrt{\left(\left(p_{1}+p_{2}+p_{3}+p_{4}\right)^{2}\right.}$, where $p_{i}$ are the four-momenta of the four leading jets. The results of the measurement are compared to NLO predictions calculated using BlackHat/Sherpa [19] and NJet/Sherpa [20]. They are 
both fixed-order calculations with no parton shower and no hadronisation. Therefore, the results are presented at the parton-jet level, that is, using jets built from partons instead of hadrons. However, the effect of non-perturbative correction in this analysis is expected to be small.

The total experimental uncertainty in these measurements is about $10 \%$, dominated by the JES and unfolding uncertainties.

The distribution of the invariant mass $m_{4 j}$ is studied in Figure 4. HEJ [21] is mostly compatible with the measurement, but the ratio to data has a bump structure in the region of approximately 1 to $2 \mathrm{TeV}$. This feature is also shared by NJet/Sherpa, but the differences with respect to the data are covered by the NLO uncertainties.

\section{Jet charge measurements in dijet events in proton-proton collisions at centre-of-mass energy $\sqrt{s}=8 \mathrm{TeV}$}

Inclusive dijet events provide an useful environment for measuring the jet charge as they are an abundant source of gluon-initiated and quarkinitiated jets. Events are required to have at least two jets with $p_{\mathrm{T}}>50 \mathrm{GeV}$ in the central region, $(|y|<2.1)$, and only the leading two jets are considered for the jet charge measurement. To select dijet topologies, the two leading jets must have $p_{\mathrm{T}}^{1} / p_{\mathrm{T}}^{2}<1.5$. Using the tracks assigned to a jet by ghost association, the jet charge $Q_{J}$ of a jet $J$ is calculated using a transverse-momentumweighting

$$
Q_{J}=\frac{1}{\left(p_{\mathrm{T}}^{J}\right)^{\kappa}} \sum_{i \in \text { Tracks }} q_{i}\left(p t^{i}\right)^{\kappa},
$$

where Tracks is the set of tracks associated with

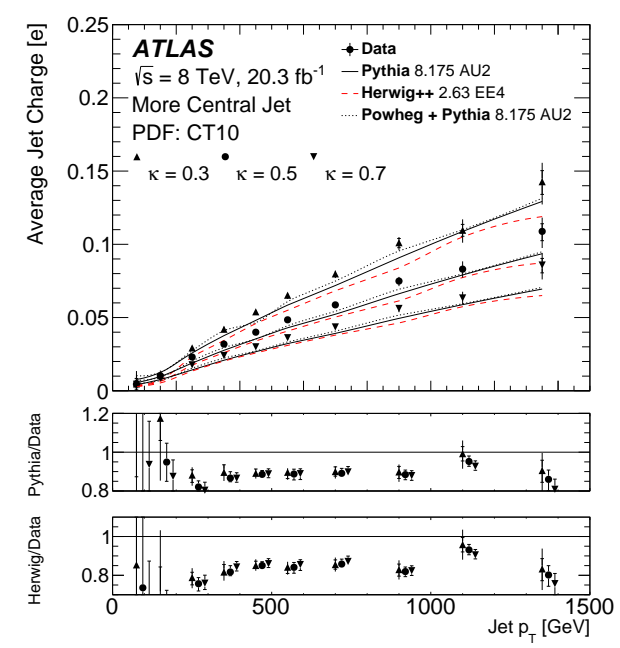

Figure 5: The measured average jet charge as a function of the jet $p_{\mathrm{T}}$ for $\kappa=0.3,0.5,0.7[10]$ using CT10 PDF set. jet $J, q_{i}$ is the charge of track $i$ with associated transverse momentum $p_{\mathrm{T}}^{i}, \kappa$ is a free regularization parameter, and $p_{\mathrm{T}}^{J}$ is the transverse momentum of the calorimeter jet.

The total experimental uncertainty in the jet charge measurements is about 3-5\%, dominated by the JES and the tracking uncertainties.

The unfolded jet charge distribution's mean is compared to NLO POWHEG+PYTHIA 8 [22] and LO PYTHIA 8 [23] theory predictions using the CT10 PDF set [18] as shown in Figure 5. The calculations are generally about $10 \%$ below the data. There does not seem to be an effect from the POWHEG NLO matrix element on the data-to-MC agreement.

\section{Inclusive jet production cross-section measurements in proton-proton collisions at centre-of-mass energy $\sqrt{s}=13 \mathrm{TeV}$}

The inclusive-jet cross-section is measured using the first data collected by the ATLAS experiment from the 2015 LHC operation at a centre-of-mass energy of $\sqrt{s}=13 \mathrm{TeV}$. The data 
correspond to an integrated luminosity of $78 \mathrm{pb}^{-1}$. The measurement is performed in a kinematic region where the performance of the ATLAS detector is well understood for the early Run-2 datataking.
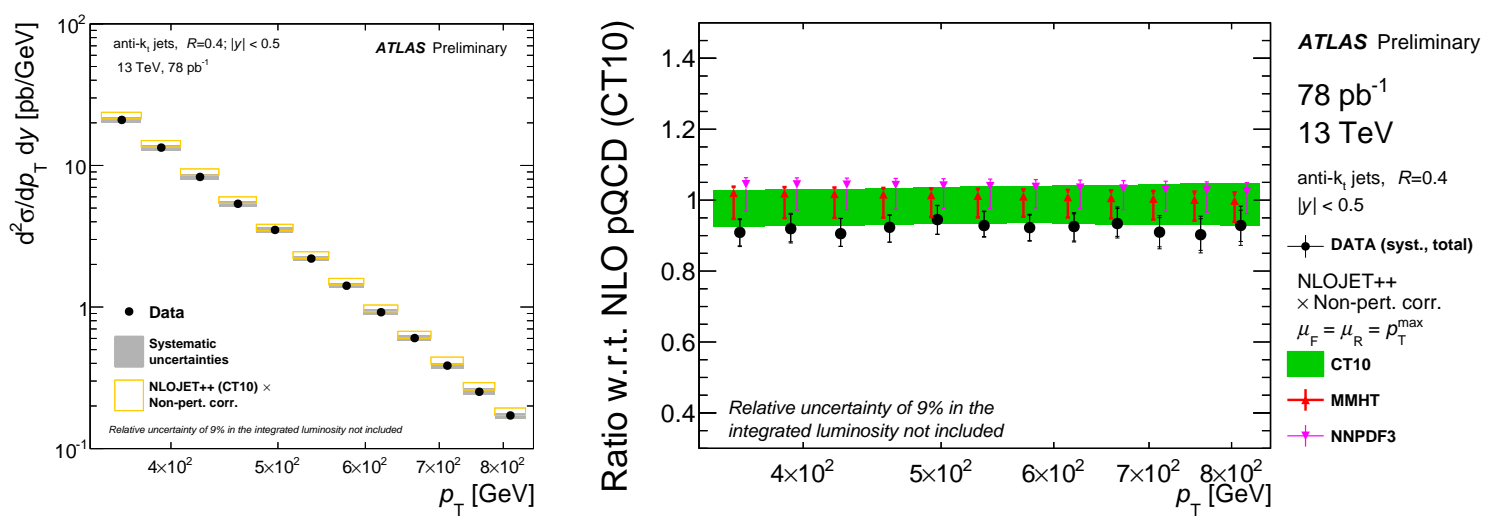

Figure 6: Inclusive-jet cross-sections [11] as a function of the jet $p_{\mathrm{T}}$ in the $|y|<0.5$ range, for anti- $k_{t}$ jets with $R=0.4$, shown in a range of $350<p_{\mathrm{T}}<840 \mathrm{GeV}$. The $\pm 9 \%$ uncertainty from the luminosity measurement is not included.

The JES calibration [24] is based on the uncertainties derived for the ATLAS data $\sqrt{s}=8 \mathrm{TeV}$ in 2012 [25, 26, 27], completed with a series of components specific to the calibration of jets in the early Run-2 data at $\sqrt{s}=13 \mathrm{TeV}$ used in this measurement.

The inclusive-jet cross-section is measured differentially as a function of the jet transverse momentum, $p_{\mathrm{T}}$, in the jet rapidity range of $|y|<0.5$ and compared to NLO QCD predictions [13] using several PDF sets and corrected for non-perturbative effects, as shown in Figure 6. The predictions are consistent with the data, and more precise measurements over a wider kinematic region are foreseen as more data are collected.

\section{Conclusions}

This contribution presents the studies of the jet production performed by the ATLAS Collaboration using datasets recorded in 2011, 2012 and 2015 at centre-of-mass energies of $\sqrt{s}=$ 7,8 and $13 \mathrm{TeV}$. These high statistics and high precision data samples allow to perform detailed quantitative tests of the Standard Model predictions. The multi-jet production cross-sections have been measured as a function of different kinematic observables probing the theoretical calculations in the $\mathrm{TeV}$ range. The jet charge measurements evaluate the evolution of nonperturbative jet observables to validate QCD MC predictions and tune their free model parameters. The transverse energy-energy correlations allows for precise $\alpha_{s}$ determination. The inclusive jet cross-section measurements using first Run-2 data test perturbative QCD at this new, unexplored energy.

\section{References}

[1] L. Evans and P. Bryant, JINST 3 (2008) S08001.

[2] M. Cacciari, G. P. Salam, and G. Soyez, JHEP 04 (2008) 063, arXiv: 0802.1189 [hep-ph] . 
[3] M. Cacciari, G. P. Salam, and G. Soyez, Eur. Phys. J. C 72 (2012) 1896, arXiv: 1111.6097 [hep-ph].

[4] ATLAS Collaboration, JHEP 11 (2013) 031, arXiv:1307.4568 [hep-ex] .

[5] ATLAS Collaboration, No. ATLAS-CONF-2013-086. Geneva, Aug, 2013. https: //cds.cern. ch/record/1572980.

[6] ATLAS Collaboration, JHEP 02 (2015) 153, arXiv: 1410.8857 [hep-ex] . [Erratum: JHEP09,141(2015)].

[7] ATLAS Collaboration, Eur. Phys. J. C 75 no. 5, (2015) 228, arXiv:1411.1855 [hep-ex] .

[8] ATLAS Collaboration, Physics Letters B 750 (2015) 427 - 447.

[9] ATLAS Collaboration, arXiv:1509.07335 [hep-ex].

[10] ATLAS Collaboration, arXiv:1509.05190 [hep-ex].

[11] ATLAS Collaboration, No. ATLAS-CONF-2015-034. Geneva, Jul, 2015. https://cds.cern.ch/record/2038145.

[12] ATLAS Collaboration, JINST 3 (2008) S08003.

[13] Z. Nagy, Phys. Rev. D 68 (2003) 094002, arXiv:hep-ph/0307268 [hep-ph] .

[14] T. Sjostrand, S. Mrenna, and P. Z. Skands, JHEP 05 (2006) 026, arXiv : hep-ph / 0603175 [hep-ph].

[15] P. Z. Skands, Phys. Rev. D82 (2010) 074018, arXiv:1005.3457 [hep-ph] .

[16] M. L. Mangano, M. Moretti, F. Piccinini, R. Pittau, and A. D. Polosa, JHEP 07 (2003) 001, arXiv:hep-ph/0206293 [hep-ph] .

[17] M. Bahr et al., Eur. Phys. J. C 58 (2008) 639-707, arXiv:0803.0883 [hep-ph] .

[18] J. Gao, M. Guzzi, J. Huston, H.-L. Lai, Z. Li, P. Nadolsky, J. Pumplin, D. Stump, and C. P. Yuan, Phys. Rev. D 89 no. 3, (2014) 033009, arXiv:1302.6246 [hep-ph] .

[19] C. F. Berger, Z. Bern, L. J. Dixon, F. Febres Cordero, D. Forde, H. Ita, D. A. Kosower, and D. Maitre, Phys. Rev. D78 (2008) 036003, arXiv:0803.4180 [hep-ph].

[20] S. Badger, B. Biedermann, P. Uwer, and V. Yundin, Comput. Phys. Commun. 184 (2013) 1981-1998, arXiv:1209.0100 [hep-ph].

[21] J. R. Andersen and J. M. Smillie, JHEP 01 (2010) 039, arXiv:0908.2786 [hep-ph] .

[22] P. Nason, JHEP 11 (2004) 040, arXiv: hep-ph/0409146 [hep-ph] .

[23] T. Sjostrand, S. Mrenna, and P. Z. Skands, Comput. Phys. Commun. 178 (2008) 852-867, arXiv:0710.3820 [hep-ph].

[24] ATLAS Collaboration, No. ATL-PHYS-PUB-2015-015. Geneva, Jul, 2015. https://cds.cern.ch/record/2037613.

[25] ATLAS Collaboration, No. ATLAS-CONF-2015-002. Geneva, Mar, 2015. https://cds.cern.ch/record/2001682.

[26] ATLAS Collaboration, No. ATLAS-CONF-2015-017. Geneva, Apr, 2015. https://cds.cern. ch/record/2008678.

[27] ATLAS Collaboration, Eur. Phys. J. C 75 (2015) 17, arXiv:1406.0076 [hep-ex]. 